\title{
Expressing the glycaemic potency of foods
}

\author{
John Monro \\ New Zealand Institute for Crop \& Food Research Ltd, Private Bag 11 600, Palmerston North, New Zealand
}

\begin{abstract}
The glycaemic index (GI) was introduced to guide food exchanges within equicarbohydrate food categories, and it expresses the glycaemic potency of the available carbohydrate component in a food relative to that of glucose. As GI is a relative value based on 'available carbohydrate' it cannot guide food choice for glycaemic control unless the foods are equal in available carbohydrate. Furthermore, GI cannot respond to food intake or to effects on food glycaemic potency of replacing glycaemic ingredients with non-glycaemic ingredients. The glycaemic glucose equivalent (GGE) overcomes these limitations of GI. The GGE content of an amount of food is the weight of glucose $(\mathrm{g})$ that would induce a glycaemic response equal to that induced by the food. Few studies have compared GI and GGE as guides to food choice for glycaemic control, but in a direct test of the predictive validity of GGE in a group of foods of differing carbohydrate and GI, GGE predicted glycaemic potency well, whereas GI was unrelated to glycaemic effect. Furthermore, an information-processing model of the use of food information in food choice shows that GI has fundamental flaws when used outside the restriction of equicarbohydrate food exchange categories. As a general guide to food choices for the control of glycaemia GI does not satisfy the criteria predictive validity, accuracy, safety, ease of use, flexibility, sufficiency and compatability, whereas GGE does. GGE is also a scientifically precise and meaningful term with which to express glycaemic potency than is 'glycaemic load'.
\end{abstract}

Carbohydrate: Glycaemic index: Glycaemic glucose equivalents: Glycaemic load

Food data for the dietary control of glycaemia have changed progressively with the growing understanding of the relationship between food properties, physiological effects and health end points. The links between postprandial glycaemia and carbohydrate intake led initially to management systems and advice based on the carbohydrate content of foods. As the magnitude of differences in the glycaemic impact of food carbohydrates became apparent, values that incorporated glycaemic potency were introduced, including glycaemic index (GI; available carbohydrate related), relative glycaemic potency (a food-based GI), glycaemic load (GL) and glycaemic glucose equivalents (GGE). The principal characteristics of the data sets are shown in Table 1 .

In the present paper expressions of glycaemic potency of foods are discussed in terms of their suitability for guiding food choices for control of postprandial glycaemia.

\section{Expressions for the glycaemic potency of carbohydrate in food}

The inadequacy of carbohydrate values alone for managing postprandial glycaemia led to development of the GI, calculated as "the area under the blood glucose response curve for each food expressed as a percentage of the area after taking the same amount of carbohydrate (as in the food) as glucose'. In an analysis of glycaemic responses to fifty-six foods Jenkins et al. (1981) demonstrated that foods within most food groups showed large differences in glycaemic effect, despite all foods being consumed at $50 \mathrm{~g}$ carbohydrate intake. The authors concluded that simple carbohydrate exchanges based on chemical analysis do not predict the physiological response to foods.

\section{Measuring glycaemic index}

Since the introduction of the GI a number of derivatives have been proposed (Table 1) and, like GI, all depend on

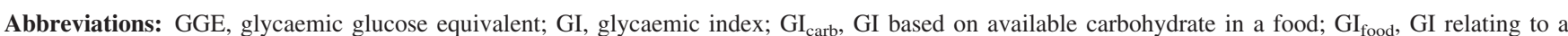
food; GL, glucose load; IAUC, incremental area under the blood glucose response curve.

Corresponding author: Dr J. A. Monro, fax +646351 7050, email monroj@crop.cri.nz
} 
Table 1. Modes of expression of the glycaemic potency of foods

\begin{tabular}{|c|c|}
\hline Basis & Definition and examples \\
\hline \multicolumn{2}{|l|}{ Glycaemic potency of food carbohydrates } \\
\hline \multirow[t]{2}{*}{$\begin{array}{l}\text { Glycaemic index } \\
\left.\text { (Gl; carbohydrate-based; } \mathrm{Gl}_{\text {carb }}\right)\end{array}$} & $\begin{array}{l}\text { GI: incremental area under the blood glucose response curve (IAUC) } \\
\text { as a result of consuming food containing } 50 \mathrm{~g} \text { available carbohydrate, as a } \\
\text { percentage of the response to } 50 \mathrm{~g} \text { glucose (Wolever et al. 1991): }\end{array}$ \\
\hline & $\mathrm{GI}_{\text {carb }}=\frac{\text { IAUC in response to food containing } 50 \mathrm{~g} \text { available carbohydrate }}{I A U C \text { in response to } 50 \mathrm{~g} \text { glucose }} \times 100$ \\
\hline \multicolumn{2}{|l|}{ Glycaemic potency of foods } \\
\hline \multirow[t]{3}{*}{$\begin{array}{l}\text { Relative glycaemic potency (RGP) } \\
\quad \text { = GI (food-based; } \mathrm{Gl}_{\text {food }} \text { ) }\end{array}$} & $\begin{array}{l}\text { RGP: glycaemic impact of a food as a percentage of the } \\
\text { effect of an equal weight of glucose (Monro, 1997, 1999): }\end{array}$ \\
\hline & $R G P=\frac{\text { IAUC in response to a relevant food portion }}{\text { IAUC in response to an equal weight of glucose }} \times 100$ \\
\hline & $\begin{array}{l}\text { RGP }=\text { GGE per } 100 \mathrm{~g} \text { food } \\
\text { RGP can be estimated as GI } \times \% \text { carbohydrate/100 }(\%)\end{array}$ \\
\hline Glycaemic glucose equivalents (GGE) & $\begin{array}{l}\text { Derived from RGP. The weight of glucose that would induce the same } \\
\text { glycaemic response as a given weight of food. (Monro \& Williams, 2000) } \\
\text { A measure of exposure to glycaemia associated with a diet (Salmerón et al. 1997) }\end{array}$ \\
\hline Glycaemic load (GL) & $\begin{array}{l}\mathrm{GL}=\sum_{\text {foods }}(\mathrm{Gl} \times \text { carbohydrate } / \text { portion } \times \text { no. of portions per } \mathrm{d} \times \text { duration of study }) \\
\text { originally based on a bread-referenced GI. Contracting GL to a single portion of a single } \\
\text { food and basing it on a glucose-referenced GI provides an estimate of GGE }\end{array}$ \\
\hline
\end{tabular}

measuring the incremental area under the blood glucose response curve (IAUC). Although there is not total agreement on the best method for measuring IAUC, the most generally accepted is the trapezoid method in which the net IAUC over the baseline value at zero time is determined by adding positive and negative trapezoids between measurement points, until the 2 or $3 \mathrm{~h}$ time limit (Wolever et al. 1991).

The value for IAUC over a period of hours does not provide a fine-grained analysis of the glycaemic response, as the same area under the curve may result from a brief high-amplitude response, or a more gradual but prolonged response. Furthermore, with prolonged glucose release the IAUC may be markedly truncated by the $2-3 \mathrm{~h}$ measurement limit. Thus, the common assumption that GI shows the rate of carbohydrate digestion may not always be true. IAUC is a coarse measure relative to the rates of physiological responses to food.

\section{Properties of glycaemic index}

During the last decade GI has been strongly promoted as a guide to food choices to minimise postprandial glycaemia (Brand-Miller et al. 2002). However, there are a number of reasons why GI may not be entirely suitable for public use, and there has certainly been a large extent of misunderstanding of GI by both the public and health professionals. A reason why GI is often misunderstood is that it is referred to as if it were a food value, whereas, by definition, it is a relative value that refers to 'available' carbohydrate in food, and not to food. Important limitations arise from the carbohydrate basis of GI:

1. if GI is to be used to directly compare foods by glycaemic potency, the foods must contain equal amounts of available carbohydrate;
2. GI is a relative value that expresses glycaemic potency as a percentage of that of glucose, so does not respond to food quantity, and cannot, therefore, be useful in predicting effects of food intakes on glycaemic response;

3. as it is defined in terms of the effect of available carbohydrate relative to an equal amount of glucose, the effect of replacing available carbohydrate in a food with a non-glycaemic or non-carbohydrate ingredient will not be reflected in the value of GI, even though the glycaemic potency of the food may be markedly reduced (Fig. 1).

\section{Expressions for the glycaemic potency of food}

In order to overcome the limitations of GI for selecting foods, glycaemic expressions have been developed to take account of the quantity of food consumed, the proportion of carbohydrate in it, as well as the glycaemic potency of the carbohydrate (Table 1).

Expressions for the glycaemic potency of food include relative glycaemic potency (Monro, 1997), GGE (Monro \& Williams, 2000) and GL (Salmerón et al. 1997). A 'relative glycaemic potency' (Table 1; Monro, 1997, 1999) value is the glycaemic effect of a food as a percentage of that of an equal quantity of glucose. In other words, it expresses the amount of glucose that would be equivalent to $100 \mathrm{~g}$ food in its glycaemic impact, i.e. GGE/100 $\mathrm{g}$ food. The GGE content of an amount of food is, therefore, the weight of glucose (g) that would induce a glycaemic response equal to that induced by the food.

Relative glycaemic potency and GGE values to date have mostly been based on GI values, not because it is best practice to do so, but because published GI values are the 


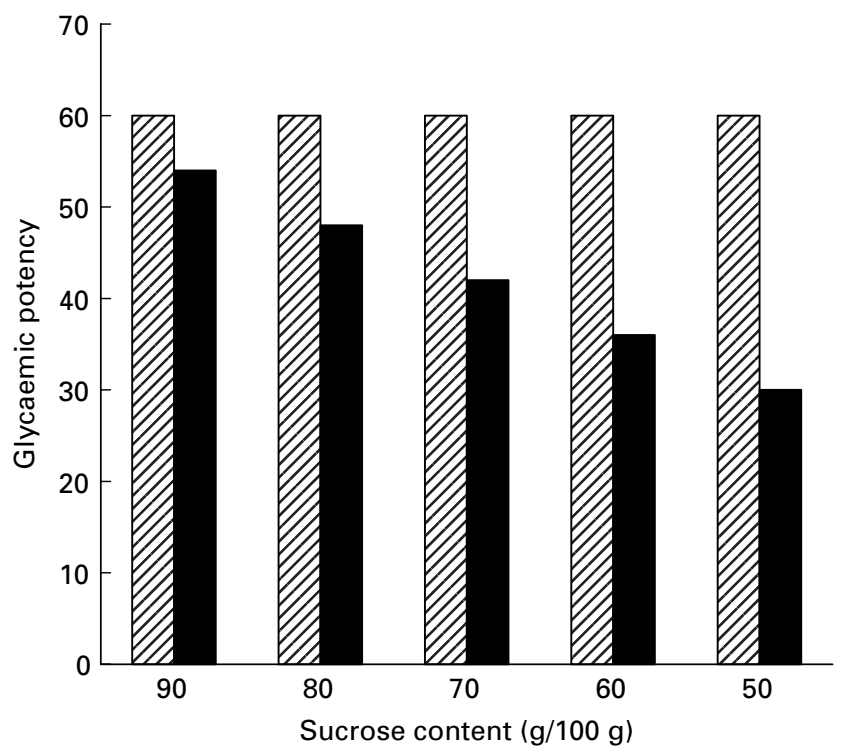

Fig. 1. Theoretical minimum effect on expressions of glycaemic potency of progressive displacement of sucrose (glycaemic index (GI) 60) from a $90 \mathrm{~g}$ sucrose $/ 100 \mathrm{~g}$ food product by a nonglycaemic food ingredient: glycaemic glucose equivalent $(/ 100 \mathrm{~g}$ food; $\mathbf{n})$, but not $\mathrm{Gl}(\square)$, shows the changes in glycaemic potency that result from sucrose substitution.

most abundant source of information on the relative glycaemic impact of foods (Foster-Powell et al. 2002). The GGE estimates derived from GI are obtained by re-expressing the glycaemic effects used to calculate GI on a food basis, rather than on an available carbohydrate basis, to obtain a 'relative glycaemic potency' value (Monro, 1997). Direct measurement and expression of the glycaemic potency of foods will provide the most accurate GGE values, because there will be no need to base the measurements on a GI value that contains errors associated with available carbohydrate values. Estimating
GGE content from GI uses the same procedure as that used for determining GL for the same weight of food in a single intake, so GL provides an approximation of true GGE.

Expressing relative glycaemic potency as a GGE content expresses glycaemic potency as if it were a food component, for which reason it has been termed a 'virtual food component' (Monro, 2004). As GGE behaves like a food component it has a number of useful properties not possessed by GI; it is responsive to food intake and, like any nutrient, it can be expressed per serving, or used for weight-to-weight comparisons of foods. Also, because it can be used to express the glycaemic potency per unit weight of food, rather than per weight of available carbohydrate, its value will reflect the effects of substituting non-glycaemic ingredients for glycaemic ingredients in food formulation.

GGE expresses a food property, glycaemic potency, without any assumptions as to the food components responsible. GI is also measured as a food effect, but it assumes that the component responsible is available carbohydrate, and its value is expressed in terms of available carbohydrate.

\section{Criteria for evaluating food data for glycaemic control}

The adequacy of GI and GGE values as guides to food choices for control of glycaemic response may be assessed using criteria based on the information-processing steps that link food information as stimuli with choosing food as a response. Intervening cognition involves the generation of goals, predictions and motor sequences that are released when goals and expected outcomes match (Fig. 2). Criteria based on the adequacy of food information to support cognitive processes underlying food choice are:

1. predictive validity: does the food value predict the outcome?

2. accuracy: are prediction and outcome (glycaemic response) close enough to allow foods with materially

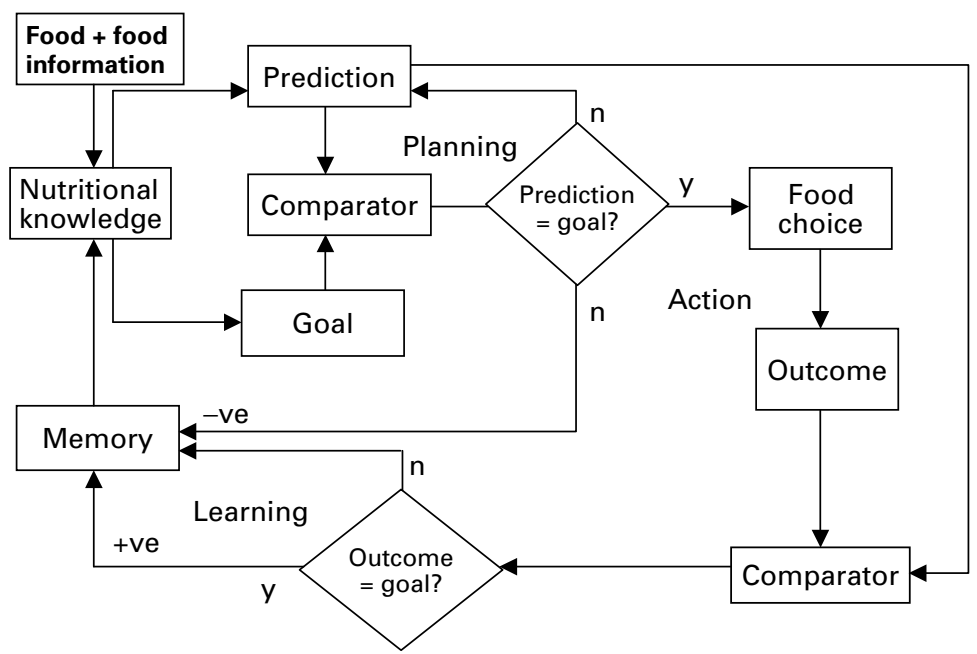

Fig. 2. Information-processing model of the role of nutritional information in food choice and in determining outcomes of food consumption. - ve, Negative; + ve, positive; n, no; y, yes. 
different effects to be distinguished by the difference in food values?

3. safety: could inaccurate food values lead to inaccurate predictions and so to food choices that diminish health?

4. ease of use: can the food value be used quickly enough and with sufficiently little effort for the consumer to be bothered with it, and get the right result?

5. understandability: is the relationship between a food value and a food effect clear; is the food value defined completely enough to make clear exactly what it represents, so that it may be used to make appropriate predictions?

6. flexibility and robustness: will the accuracy of prediction allowed by a food value hold with differing food intakes, differing food compositions and across differing food groups?

7. sufficiency: are enough of the variables that determine glycaemic response incorporated into the food value to allow it to be used directly as a predictor of glycaemic response? Do potential users of the food value have sufficient nutritional knowledge to set nutritional goals that the food value should help them to achieve?

8. compatability: is the food value in a form that will not lead to error when the consistency of cognitive processing leads users to treat it in the same way as they would other nutritional information with which it is associated in a food label?

\section{Evaluating glycaemic index and glycaemic glucose equivalent as glycaemic expressions for food choice}

These fundamental properties of effective food information provide criteria with which to compare the suitability of GI and GGE as guides to food choices for control of the glycaemic response.

\section{Predictive validity}

A relationship between both GI and GL and health end points has been demonstrated in a number of clinical and epidemiological studies (Augustin et al. 2002). Experimental clinical tests of GI usually hold carbohydrate intake constant to ensure that the influence of GI is not confounded by carbohydrate intake. On the other hand, epidemiological associations between GI or GL and end points such as heart disease, show the possible importance of an overall exposure to glycaemia, or of the average GI or GL of a combination of foods in a diet over a period of time (Salmerón et al. 1997), but do not refer to the predictive validity for choosing individual foods.

In contrast, in the context of control of glycaemia through food choice an appropriate test of validity is whether or not the GI or GGE value predicts the effects of individual food choices on postprandial glycaemia under conditions in which foods are normally selected and consumed. Under usual conditions of food use it is not possible to accurately standardise food composition and quality, or constrain selections to within homogeneous food categories.
Only two studies of GGE (GL) have been conducted in which food intake has been varied, and they have both shown that GGE predicts blood glucose response, and that the relationship is approximately linear in the normal range of carbohydrate intakes (Brand-Miller et al. 2003; Liu et al. 2003). However, studies that demonstrate a link between GI and postprandial glycaemia under equicarbohydrate conditions also support the validity of GGE, because GI is simply a special case of GGE in which foods are compared at an equal carbohydrate dose.

Simply by examining the definition of GI it can be seen that GI cannot possibly be a valid predictor of the glycaemic impact of foods in general, because it is based on a component of foods, available carbohydrate, and not on foods as such. Thus, replacing a proportion of available carbohydrate in a food by a non-available ingredient, such as isomaltose or resistant starch, may materially lower the glycaemic potency of a food but have no effect on the GI value, because GI refers only to the available carbohydrate component.

GI cannot be a reliable guide to food choice for glycaemic control under the conditions in which individuals normally choose foods, whereas the limited evidence to date suggests that GGE could be reliable.

\section{Accuracy}

The accuracy with which GI and GGE (GL) are measured and the accuracy of food choice that they confer are both important issues that are related to validity. Concern has often been expressed about the variability intrinsic to GI measurements (Pi-Sunyer, 2002) and, therefore, to GGE or GL determinations based on GI. Intra-subject variation is high, and the inaccuracy of available carbohydrate determination, particularly when carbohydrate by difference is used to calculate food allocation for GI measurement, adds to the inaccuracy.

In the context of food choice, accuracy refers to the exactness with which a food value provided for the control of glycaemia can predict glycaemic response under normal conditions for using food data. It is important to public health that food values predict outcomes, because if they do not, the values will be cognitively tagged as unsuccessful and rejected in future planning.

GI is suitable for use within a carbohydrate exchange system in which foods of similar composition are compared. When exchanges are extended to less-homogeneous categories such as breads or breakfast cereals, in which foods are grouped by culinary characteristics rather than by similarity of composition, accuracy is reduced. Inaccuracy becomes considerable when GI is applied across food groups, or to the heterogeneous groups of foods typical of many diets and differing greatly in intake and composition, such as fruits, vegetables and pulses (Monro, 2001). GGE is not subject to an equicarbohydrate restriction, and can be used to compare any quantities of foods, within reason, whether or not they are equal in carbohydrate or in the same food grouping.

The effect of differing portion sizes and carbohydrate contents of foods on the accuracy with which GGE and GI are able to predict glycaemic response is shown in 


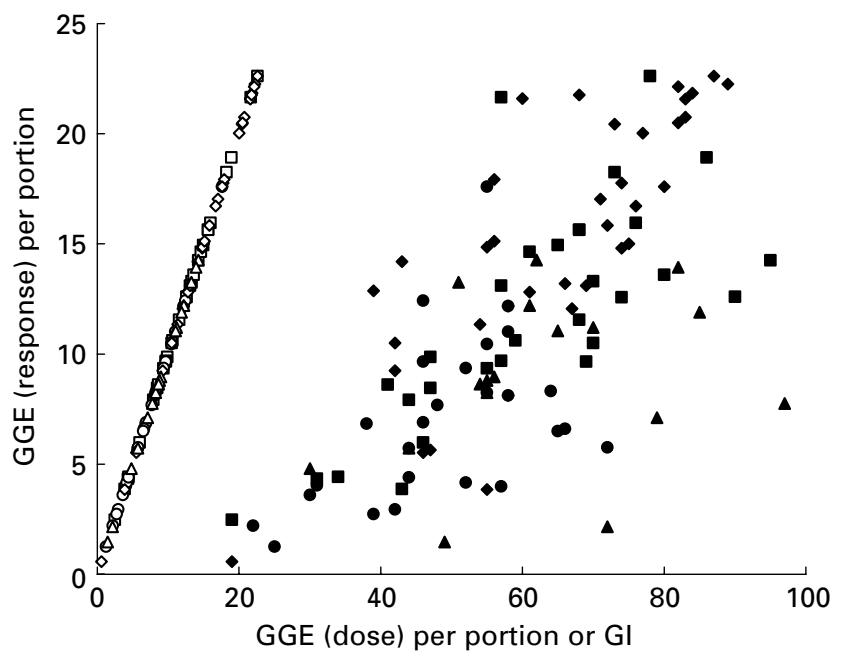

Fig. 3. Best possible prediction of glycaemic response using glycaemic glucose equivalent (GGE) values based on glycaemic responses to foods, and glycaemic index (GI) values based on glycaemic responses to carbohydrate in foods. ( $\square, \mathbf{\square}$ ), Breads; $(\diamond, \diamond)$, breakfast cereals; $(\bigcirc, \bullet)$, fruit; $(\triangle, \boldsymbol{\Delta})$, vegetables; $(\square$, $\diamond, \bigcirc, \triangle)$, GGE values; ( $\mathbf{\bullet}, \bullet, \bullet, \mathbf{\Delta})$, Gl values. GGE (response) $=$ individual incremental area under the blood glucose response curve (IAUC) to GGE dose/typical IAUC per GGE for the individual.

Fig. 3, in which values for GGE intake and GI for breads, breakfast cereals, fruit and vegetables are plotted $v$. theoretical glycaemic response, to give a best possible prediction of glycaemic response by GGE and GI. Although Fig. 3 is based on literature values, the validity of GGE dose as a predictor of response also expressed as glucose equivalents has been tested in a clinical trial (Liu et al. 2003), with twelve subjects with type 2 diabetes and twelve subjects who did not have diabetes. Using the relationship:

\section{GGE (response)}

$=\frac{\text { individual IAUC to calculated GGE dose (prediction) }}{\text { typical IAUC per GGE for the individual }}$,

the GGE dose predicted the response expressed as GGE in twenty-one of twenty-four intakes (two doses $\times$ six foods $\times$ two subject groups) with an overall relationship between prediction and response of $R^{2} 0.88$ for the whole data set.

The conclusion to be drawn from Fig. 3 is that, although GI may function well for predicting the relative effects of equal weights of carbohydrates in foods, it is not appropriate to use it to discriminate between foods varying in portion size and carbohydrate content, whereas the accuracy of GGE extends beyond equal carbohydrate comparisons to foods as a whole.

\section{Safety}

Differences in the carbohydrate content and portion size of foods, even within food groups such as breads and breakfast cereals, cause enough overlap in high-, medium- and low-GI categories for it to be possible to exchange a portion of a high-GI product for a low-GI product and substantially increase glycaemic impact (Monro, 2001). As individuals are habitual in their use of foods, and the longterm effects of hyperglycaemia and hyperinsulinaemia are damaging to health, increases in glycaemic response as a result of incorrect food classification are potentially harmful.

To ensure safety, it is important that an expression of glycaemic potency for food choice should indicate the effect of the food, not that of a food component.

Thus, GI is potentially unsafe, whereas GGE is safe because it accounts for the effects of food composition and portion size.

\section{Ease of use}

GI is often said to be easy to use, and it would be easy to use if it represented the glycaemic potency of food rather than of the available carbohydrate in food. When confronted with the usual array of foods differing in composition and eaten in different portion sizes, the consumer needs to adjust GI for carbohydrate content and food intake to be able to gauge the relative glycaemic effect of a food. In a GGE value the carbohydrate content and food quantity are already taken into account.

GGE is, therefore, easier to use correctly than GI, in most food-choice situations.

\section{Understandability}

The indirectness of the link between a value for GI and glycaemic response is not understood by most consumers and food writers, or by many health professionals. A common belief is that GI ranks foods according to glycaemic impact, whereas it ranks foods by the relative glycaemic potency of their available carbohydrate component, so that the cognitive predictions that lead to food choices may be quite incorrect when based on GI.

A food variable, such as GGE, that directly represents the glycaemic potency of a portion of food, also allows comparison of equal portions and shows the effect of food intake will make more sense than a static value such as GI that does not represent the glycaemic impact of food intake or composition.

GGE is, therefore, much more understandable than GI.

\section{Flexibility and robustness}

Flexibility and robustness refer to the range of situations in which a food value can be relied on to make an accurate prediction of blood glucose effects.

GI is intrinsically inflexible because it is expressed as the effect of a $50 \mathrm{~g}$ carbohydrate dose relative to $50 \mathrm{~g}$ glucose. As a relative value, GI does not change with carbohydrate content or with food intake, yet both have a large impact on glycaemic response. By incorporating food composition and intake, GGE is a flexible value that may be applied across different food groups and intakes.

A few studies have investigated the relationship between food intake and the prediction of glycaemic response by GGE (GL). Several foods differing in GI and carbohydrate content and fed at the same GGE dose give similar 
responses, and doubling the GGE dose doubles the response (Liu et al. 2003). There is no marked relationship between carbohydrate intake and the response per GGE with a food intake of $\leq 60 \mathrm{~g}$, but at an intake of $>100 \mathrm{~g}$ carbohydrate in noodles the response per GGE is suppressed by about $10 \%$. In a similar study Brand-Miller et al. (2003) have shown that increments in GL lead to increments in glycaemic response, and that the response is a linear function of GL up to intakes of $>100 \mathrm{~g}$ carbohydrate. Wolever \& Bolognesi (1996) have also reported a linear relationship between food intake and glycaemic response, with a plateau at carbohydrate intakes $>50 \mathrm{~g}$. However, calculations from the data of Wolever \& Bolognesi (1996) show that the glycaemic response is close to a linear function of intake between about 25 and $100 \mathrm{~g}$ carbohydrate intake for most samples, and that plateau effects may be partly associated with truncation of the IAUC at the $2 \mathrm{~h}$ cut-off.

When non-carbohydrate components of foods, including water, are taken into account the amount of food that must be consumed to approach $100 \mathrm{~g}$ carbohydrate intake is often found to be quite large (Monro, 1999). Thus, from the data available so far it seems that in relation to variations in carbohydrate dose and food intake GGE (GL) is quite robust, in the sense that it accurately reflects the changes in glycaemic impact that would arise from usual variations in carbohydrate and food intakes. GI, on the other hand, is a fixed value that does not change with food or carbohydrate intake.

\section{Sufficiency}

GI must be combined with the available carbohydrate content and intake of a food to produce an estimated GGE value that will predict relative glycaemic impact accurately (Fig. 2). GGE values have been designed with a specific aim of being sufficient to predict relative glycaemic effect.

Thus, GI is not sufficient to predict the relative glycaemic effect of a food, but GGE is sufficient.

\section{Compatibility}

As there is a cognitive tendency to treat apparently similar information in a consistent manner, expressions of glycaemic potency should be compatible with, and act consistently with, existing knowledge or experiences with food and food information, or they are likely to be used incorrectly.

Misunderstanding of GI arises from the natural assumption that it behaves in the same way as similar information, such as nutrient values, on the same food packet; however, it does not. Unlike nutrient values, which refer to food, GI refers to the available carbohydrate component in food. In contrast, GGE acts in the same way as a food component; it is compatible with most other information given in nutrient information panels and can be expressed in the same way. GGE responds to food intake as a nutrient is expected to respond (Monro \& Williams, 2000).

Compatibility is important in applying GGE to nutritional management. GGE, as a virtual food component, can be used alongside nutrient information to concurrently show the effects of food consumption on nutrient intake and relative glycaemic effect, to provide a more complete view of a food effect than is provided by nutrient values alone.

The conclusion is that GGE is more compatible than GI with other food information used in choosing foods.

\section{Terminology of glycaemic expression}

Terms develop meaning through use, but in a scientific context the words from which a term is constructed should convey meaning as precisely as possible.

An index is defined in the Oxford English Dictionary (Simpson \& Weiner, 1989) as '- a number expressing a physical property etc. in terms of a standard'. A 'GI of a food' should express the glycaemic potency of a food in terms of a reference. As currently used, GI is inappropriately defined, as there is no information intrinsic to the term to specify that the test material is not food but available carbohydrate, or that the reference is glucose. The lack of information conveyed by the term 'GI' and the usual reference to it as the 'GI of a food' (Brand-Miller et al. 2002) is partly responsible for the widespread misunderstanding of GI.

Thus, the GI currently in use (GI based on the available carbohydrate; $\mathrm{GI}_{\mathrm{carb}}$ ) should be defined as expressing the glycaemic potency of the food carbohydrate, and the 'GI of a food' (GI based on the food; $\mathrm{GI}_{\text {food }}$ ) should express the glycaemic potency of the food relative to glucose. In fact, both $\mathrm{GI}_{\text {carb }}$ and $\mathrm{GI}_{\text {food }}$ are based on food effects, but the determination and calculation of a $\mathrm{GI}_{\text {carb }}$ value is arbitrarily based on the nominal glycaemic carbohydrate content of a food. The actual available carbohydrate content on which GI is based is, in fact, seldom measured.

\section{Glycaemic load or glycaemic glucose equivalents?}

The meaning of GL has drifted from the time of its original use as a measure of exposure to glycaemia in epidemiological studies (Salmerón et al. 1997). Applied to a single serving of a food and calculated from a glucosereferenced GI value, the GL of a food quantity consumed at a specific time is a numerical approximation to GGE content. The term 'GL' has little intrinsic meaning other than that it is related to glycaemia. The expression ' $\mathrm{GL}=8$ ' gives no indication of what the number refers to or what the load consists of, and would not be permitted in a nutrient information panel.

In contrast, GGE is a term that has been designed to have meaning. If a food label for a $65 \mathrm{~g}$ muesli bar states 'GGE (g) 8' it is spelling out clearly that the $65 \mathrm{~g}$ muesli bar would have a glycaemic impact equivalent to that of $8 \mathrm{~g}$ glucose, and is expressed in exactly the same way as a nutrient. GGE could therefore serve as the unit required to give GL meaning.

\section{A practical food glycaemic index that links glycaemic impact to food intake}

As described in Table 1, GGE is derived from the foodbased GI, the relative glycaemic potency, which may be alternatively expressed as $\mathrm{GGE} / 100 \mathrm{~g}$ food, or $\mathrm{GI}_{\text {food }}$ 


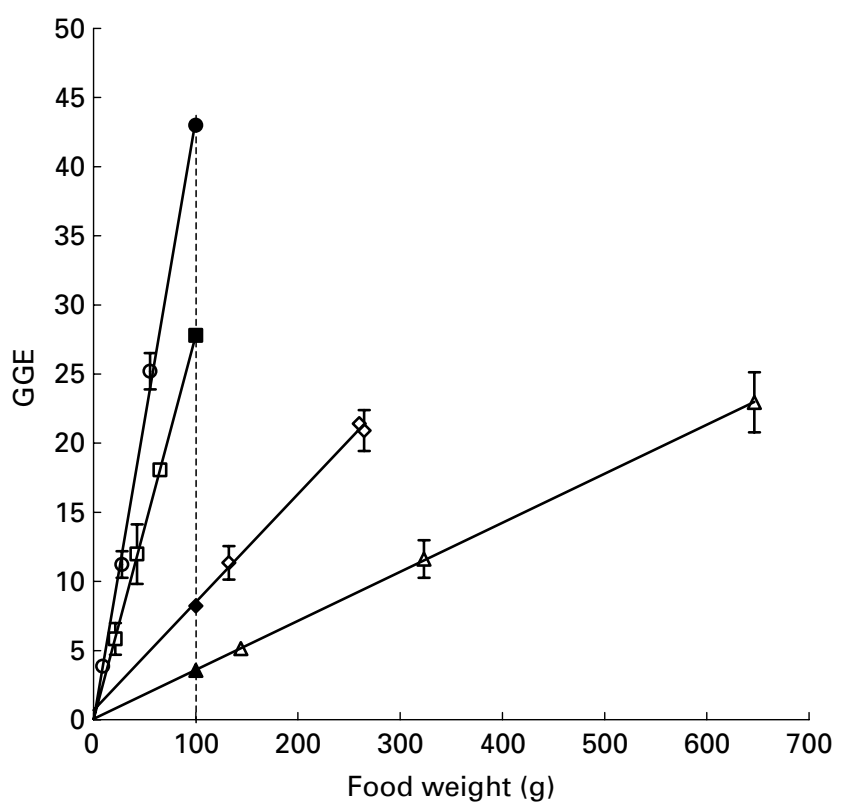

Fig. 4. The relationship between the relative glycaemic potency of food (food glycaemic index, which is the glycaemic glucose equivalent $(\mathrm{GGE}) / 100 \mathrm{~g}(\bullet, \mathbf{\square}, \bullet, \mathbf{\Delta}))$, GGE per serving $(\bigcirc, \square$, $\triangle, \diamond$; values with no error bars) and the dose-response relationship between GGE intake and glycaemic response expressed as GGE (-) for biscuits $(\bigcirc)$, rice ( $\square$; dry weight), yam $(\triangle)$ and porridge $(\diamond) .(\bigcirc, \square, \triangle, \diamond)$, Experimentally-determined values are shown as means with their standard errors represented by vertical bars for twelve subjects per determination. (Based on data in Liu et al. 2003.)

(Monro, 2003). As indices, values for GGE/100 g food would allow a direct comparison of the glycaemic potency of foods on a weight-for-weight basis, which is what is currently attempted inappropriately with $\mathrm{GI}_{\text {carb }}$. However, if a carbohydrate-related measure of glycaemic potency of foods equivalent to $\mathrm{GI}_{\text {carb }}$ were required, it could be achieved as the GGE content of foods containing the same amount of carbohydrate.

Being able to express a food GI as GGE/100 $\mathrm{g}$ food allows the estimation of the glycaemic impact of any quantity of food, within reason. It means that the GGE content per $100 \mathrm{~g}$ and per serving will both lie on the same food dose-glycaemic response line. Fig. 4 shows the relationship between $\mathrm{GI}_{\text {food }}$ (GGE/100 g), GGE per common standard measure and the line that relates GGE response to food intake, and it shows how useful values would be for choosing foods if they were based on the glycaemic potency of foods.

A hypothetical nutrient information panel (Table 2) shows how GGE might be used as a virtual food component to accurately express the glycaemic potency of a serving and of $100 \mathrm{~g}$ of the food, in a format compatible with other food information.

\section{Conclusion}

GI is a measure of the relative glycaemic potency of food carbohydrates designed for use in equicarbohydrate
Table 2. Glycaemic glucose equivalent as a virtual food component expressing relative glycaemic impact in terms of the weight of glucose causing a glycaemic response equivalent to given food weights; data for muesli, serving size approximately $65 \mathrm{~g}$

\begin{tabular}{lcc}
\hline & Per $65 \mathrm{~g}$ & Per $100 \mathrm{~g}$ \\
\hline Energy (kJ) & 1040 & 1600 \\
Protein (g) & $5 \cdot 9$ & $9 \cdot 1$ \\
Fat $(\mathrm{g})$ & $5 \cdot 1$ & $7 \cdot 8$ \\
Total carbohydrate $(\mathrm{g})$ & 46 & 70 \\
Total niacin equivalent (mg) & $1 \cdot 6$ & $2 \cdot 5$ \\
Glycaemic glucose equivalent $(\mathrm{g})$ & 18 & 28 \\
\hline
\end{tabular}

exchanges. Outside equicarbohydrate categories, in an environment containing variations in food composition and portion size that typify most diets, GI does not satisfy the criteria (predictive validity, accuracy, safety, ease of use, flexibility, sufficiency and compatibility), whereas GGE does.

Modes of expression of glycaemic potency that will assist food choices for glycaemic control need to be based on food rather than on the available carbohydrate of food. GGE and GL are expressions of the glycaemic potency of food. GGE is a scientifically credible term that is expressed similarly to other nutrients in nutrient information panels. Although GL is numerically the same as GGE, it has little meaning as an expression and it has no units, unless it is used as an expression for the total GGE content in a food quantity.

If GGE/100 g were to be used to allow weight-forweight comparisons of foods, and GGE per serving were to be used to indicate the relative glycaemic potency of servings, glycaemic potency would be expressed as a virtual food component as complete as, and consistent with, other values in nutrient information panels, and it would indicate the effect of non-available carbohydrate ingredients on the glycaemic potency of foods. GI, which has been difficult for consumers to use correctly, could then be dispensed with as a guide to food choice for the control of postprandial glycaemia.

\section{Acknowledgement}

The generosity of Palatinit $\mathrm{GmbH}$ for sponsorship is gratefully acknowledged.

\section{References}

Augustin LS, Franceschi S, Jenkins DJA, Kendall CWC \& La Vecchia C (2002) Glycemic index in chronic disease: a review. European Journal of Clinical Nutrition 56, 1049-1071.

Brand-Miller J, Foster-Powell K \& Clagiuri S (2002) The New Glucose Revolution. Sydney, New South Wales: Hodder Headline Australia Pty Ltd.

Brand-Miller JC, Thomas M, Swan V, Ahmad ZI, Petocz P \& Colagiuri S (2003) Physiological validation of the concept of glycemic load in lean young adults. Journal of Nutrition 133, 2728-2732.

Foster-Powell K, Holt SHA \& Brand-Miller JC (2002) International table of glycemic index and glycemic load values: 2002. American Journal of Clinical Nutrition 76, 5-56. 
Jenkins DJ, Wolever TM, Taylor RH, Barker H, Fielden H, Baldwin JM, Bowling AC, Newman HC, Jenkins AL \& Goff DV (1981) Glycemic index of foods: a physiological basis for carbohydrate exchange. American Journal of Clinical Nutrition 34, 362-366.

Liu P, Perry T \& Monro JA (2003) Glycaemic glucose equivalent: validation as a predictor of the relative glycaemic effect of foods. European Journal of Clinical Nutrition 57, 1141-1149.

Monro JA (1997) Glycaemic index and available carbohydrates in exchanges of New Zealand foods. Proceedings of the Nutrition Society of New Zealand 22, 241-248.

Monro JA (1999) Available carbohydrate and glycemic index combined in new data sets for managing glycemia and diabetes. Journal of Food Composition and Analysis 12, 71-82.

Monro JA (2001) Glycaemic index-based classifications do not reflect the relative glycemic impact of foods. Proceedings of the Nutrition Society of New Zealand 24, 96-103.

Monro JA (2003) Redefining the glycemic index for dietary management of postprandial glycemia. Journal of Nutrition 133, 4256-4258.
Monro JA (2004) Virtual food components: functional food effects expressed as food components. European Journal of Clinical Nutrition 58, 219-230.

Monro JA \& Williams M (2000) Concurrent management of postprandial glycaemia and nutrient intake using glycaemic glucose equivalents, food composition data, and computerassisted meal design. Asia Pacific Journal of Clinical Nutrition 9, 67-73.

Pi-Sunyer FX (2002) Glycemic index and disease. American Journal of Clinical Nutrition 76, 290S-298S.

Salmerón J, Manson JE, Stampfer MJ, Colditz GA, Wing AL \& Willet WC (1997) Dietary fiber, glycemic load, and risk of non-insulin-dependent diabetes mellitus in women. Journal of the American Medical Association 277, 472-477.

Simpson J \& Weiner E (editors) (1989) Oxford English Dictionary, 3rd ed. Oxford: Oxford University Press.

Wolever TMS \& Bolognesi C (1996) Source and amount of carbohydrate affect postprandial glucose and insulin in normal subjects. Journal of Nutrition 126, 2798-2806.

Wolever TMS, Jenkins DJA, Jenkins AL \& Josse RG (1991) The glycemic index: methodology and clinical implications. American Journal of Clinical Nutrition 54, 846-854. 